\title{
Research
}

Amandeep Khatter, Frank Moriarty, Mark Ashworth, Stevo Durbaba and Patrick Redmond

\section{Prevalence and predictors of potentially inappropriate prescribing in middle-aged adults:}

a repeated cross-sectional study

\begin{abstract}
\section{Background}

Potentially inappropriate prescribing (PIP) is common in older adults and known to be associated with polypharmacy and multimorbidity. Less is known about the prevalence and causes of $\mathrm{PIP}$ in middle-aged adults.
\end{abstract}

\section{Aim}

To determine the prevalence and predictors of PIP in middle-aged adults.

\section{Design and setting}

A repeated cross-sectional study was conducted using primary care data in London.

\section{Method}

PIP was defined using the PRescribing Optimally in Middle-aged People's Treatments (PROMPT) criteria. Prescribing and demographic data were extracted from Lambeth DataNet (LDN). a pseudonymised database of all patients registered at general practices in Lambeth, for those aged 45-64 years prescribed $\geq 1$ medicines in each year from 2014-2019 ( $n=46$ 633-52 582). Prevalence and trends over 6 years were investigated, including the association of PIP with polypharmacy, multimorbidity, deprivation, sex, and age.

\section{Results}

The prevalence of PIP decreased from $20 \%$ in 2014 to $18 \%$ in 2019. The most prevalent PROMP criteria in 2019 were the use of $\geq 2$ drugs from the same pharmacological class (7.6\%), use of nonsteroidal anti-inflammatory drugs for $>3$ months $(7.1 \%)$ and use of proton pump inhibitors above recommended maintenance dosages for $>8$ weeks (3.1\%). Over the study period, the prevalence of multimorbidity increased (47-52\%) and polypharmacy remained stable (27\%). Polypharmacy, multimorbidity, deprivation, and age were independently associated with PIP. Sex was the only variable not associated with PIP.

\section{Conclusion}

Almost one-fifth of middle-aged adults prescribed medicines are exposed to PIP, as defined by the PROMPT criteria. This is likely to be linked with exposure to avoidable adverse drug events. The PROMPT criteria may provide a useful aid in interventions to optimise prescribing.

\section{Keywords}

anti-inflammatory agents; drug-related side effects and adverse reactions; general practice; inappropriate prescribing; middle aged; multimorbidity; non-steroidal; polypharmacy.

\section{INTRODUCTION}

Potentially inappropriate prescribing (PIP) involves using medicines that may cause more harm than benefit, are not costeffective, or are not clinically indicated. Increased hospitalisation, adverse drug events (ADEs), and emergency department visits have been reported as detrimental effects of PIP. PIP is costly; costs are incurred through the use of unnecessary medicines or additional healthcare utilisation. $^{3} \quad$ High-risk prescribing (prescriptions most likely to cause ADEs) also varies between prescribers, with differences reported between general practices and between individual GPs. ${ }^{4}$

PIP in older adults laged 265 ) has been extensively studied, with possibly $20 \%$ of prescriptions given to older adults categorised as potentially inappropriate. ${ }^{5}$ Polypharmacy (commonly defined as taking $\geq 4$ medicines daily) and multimorbidity lcommonly defined as having $>1$ longterm condition [LTC]) are strongly associated with PIP in older adults. ${ }^{6-10}$ However, multimorbidity is not confined to older adults, with $30 \%$ of middleaged (45-64 years) adults also having multimorbidity. ${ }^{11}$ Indeed, in absolute terms, there are more people with multimorbidity aged under 65 than over. ${ }^{11}$ Similarly, Cooper and others ${ }^{12}$ found that, in 2012, 20\% of

A Khatter, BSc (Hons), medical student; M Ashworth, BSc (Pharm), DM, MRCP, FRCGP reader in primary care; $\mathbf{S}$ Durbaba, BEng. MSc, database manager, School of Population Health and Environmental Sciences, King's College London, London, UK. F Moriarty, BSc (Pharm), MPharm, PhD, lecturer in pharmacy, School of Pharmacy and Biomolecular Sciences P Redmond, PhD, MRCPI, MICGP, FHEA, senior lecturer in general practice, Department of General Practice, Royal College of Surgeons in Ireland, Dublin, Ireland.

Address for correspondence

Amandeep Khatter, School of Population Health middle-aged adults in Northern Ireland experienced polypharmacy.

Despite the prevalence of multimorbidity and polypharmacy, there has been limited research on the prevalence of PIP within middle-aged adults. The PRescribing Optimally in Middle-aged People's Treatments (PROMPT) criteria were developed to estimate the prevalence of PIP in this age group (see Supplementary Table S1 for details). ${ }^{13}$ PROMPT specifies 22 criteria for PIP, covering a broad range of drug classes, which includes a broad range of human physiological systems, as well as duplications in drug classes, for example, strong opioids should not be prescribed without the concurrent prescription of a laxative. $^{13}$

Previous studies have suggested that the prevalence of PIP using PROMPT ranges from $21.1-42.9 \%$, with increased rates associated with polypharmacy, age, multimorbidity, and female sex. ${ }^{12,14-16}$ However, these studies were crosssectional and drawn from selected population groups. There have been no longitudinal studies completed using PROMPT in the UK.

The aim of this study was to measure the prevalence and potential predictors of PIP defined using the PROMPT criteria in middle-aged adults.

and Environmental Sciences, Guy's Campus, King's College London, London SE1 1UL, UK

Email: amandeep.khatterakcl.ac.uk

Submitted: 23 November 2020; Editor's response: 6 January 2021; final acceptance: 12 February 2021

\section{(c)The Authors}

This is the full-length article (published online XXXXX 2021) of an abridged version published in print. Cite this version as: $\mathbf{B r} \mathbf{J}$ Gen Pract 2021; DOI: https://doi.org/10.3399/BJGP.2020.1048 


\section{How this fits in}

Potentially inappropriate prescribing (PIP) can lead to adverse drug events, as well as increased hospitalisation and healthcare costs. There is limited research on the prevalence and predictors of PIP in middle-aged adults. This study found that PIP is not confined to older adults, and is common in middle-aged adults too. It is also more likely in older, socioeconomically deprived patients, as well as those with polypharmacy and multimorbidity. These findings will help GPs to identify patients at increased risk of PIP in middle age.

\section{METHOD}

\section{Study design and setting}

A repeated cross-sectional study was conducted using general practice-derived data from Lambeth DataNet (LDN) and reported as per the STrengthening the Reporting of OBservational studies in Epidemiology (STROBE) checklist. ${ }^{17}$ LDN contains the pseudonymised patient records of all 41 general practices in Lambeth Clinical Commissioning Group (1 185335 patients aged $\geq 18$, excluding the $3.8 \%$ of patients who have opted out of data sharingl and has been extensively used in database research. ${ }^{18,19}$ The data used were confined to patients prescribed $\geq 1$ medicine and aged 45-64 years in each of the years 2014-2019 inclusive. Demographically, $65 \%$ of Lambeth's population are socioeconomically deprived (deprivation score in the bottom two quintiles), 52\% are female, and over half belong to a white ethnic group.

\section{Outcomes}

The primary outcome was the prevalence and types of PIP as described by the PROMPT criteria. PIP was explored as both a dichotomous and count variable. The secondary outcome was the association of the variables age, sex, multimorbidity, polypharmacy, and deprivation with both the presence of PIP (binary variable) and with a count of PIP over the 6 years.

\section{Covariates}

The models were adjusted for important covariates identified a priori from literature. Age was explored in the regression models as a categorical variable. Multimorbidity was defined as those with $\geq 2$ of 32 LTCs (see Supplementary Box S1 for details). This definition was derived from Cassell and others ${ }^{20}$ codes and modified following local consultation (hence inclusion of sickle cell disease and lupus). For Quality and Outcomes Framework (QOF) conditions, the study accepted QOF definitions of Read and Systematized Nomenclature of Medicine Clinical Terms (SNOMED-CT). For the conditions that rely on medication code (for example, chronic pain) Egton Medical Information Systems codes were used (data available on reasonable request). The definition of polypharmacy, used as a count variable rather than implying appropriateness, was those prescribed $\geq 4$ repeat medicines in a year. 6 Sex was included as a binary variable. Deprivation was analysed as the locally determined Index of Multiple Deprivation quintile, which is assigned based on lower layer super output areas of residence of each patient. The distribution of individuals in the study population was analysed by each of these variables and PIP.

\section{Analysis}

Descriptive statistics were used to describe the prevalence of PIP in the study population. The percentages calculated for each of the PROMPT criteria represented the study population prevalence.

Unadjusted and adjusted logistic regression was performed to assess the association of PIP with study year (using 2014 as the reference year), age group, sex, deprivation, multimorbidity, and polypharmacy. A cluster variable incorporating the patient ID with the code for their general practice was created to allow for intraclass correlation and was included in the regression model. Odds ratios with $95 \%$ confidence intervals (CI) are presented.

Negative binomial regression was used to quantify the change in the rate of PIP associated with the included covariates. Incidence rate ratios (IRR) with $95 \% \mathrm{Cl}$ are presented. A negative binomial model was used over a Poisson model owing to overdispersion of PIP rates.

Data were checked for non-random missingness with no imputation required. Regression diagnostics were run to ensure goodness of fit. All statistical analyses were carried out using Stata 14.

\section{RESULTS}

\section{Descriptive}

The number of individuals included in this study ranged from 46633 in 2014 to 52582 in 2019. Table 1 shows the descriptive statistics for the population. The study population was positively skewed towards the younger age categories, with 32\% aged 45-49 and $17 \%$ aged $60-64$. Similarly, there 
Table 1. Study population characteristics

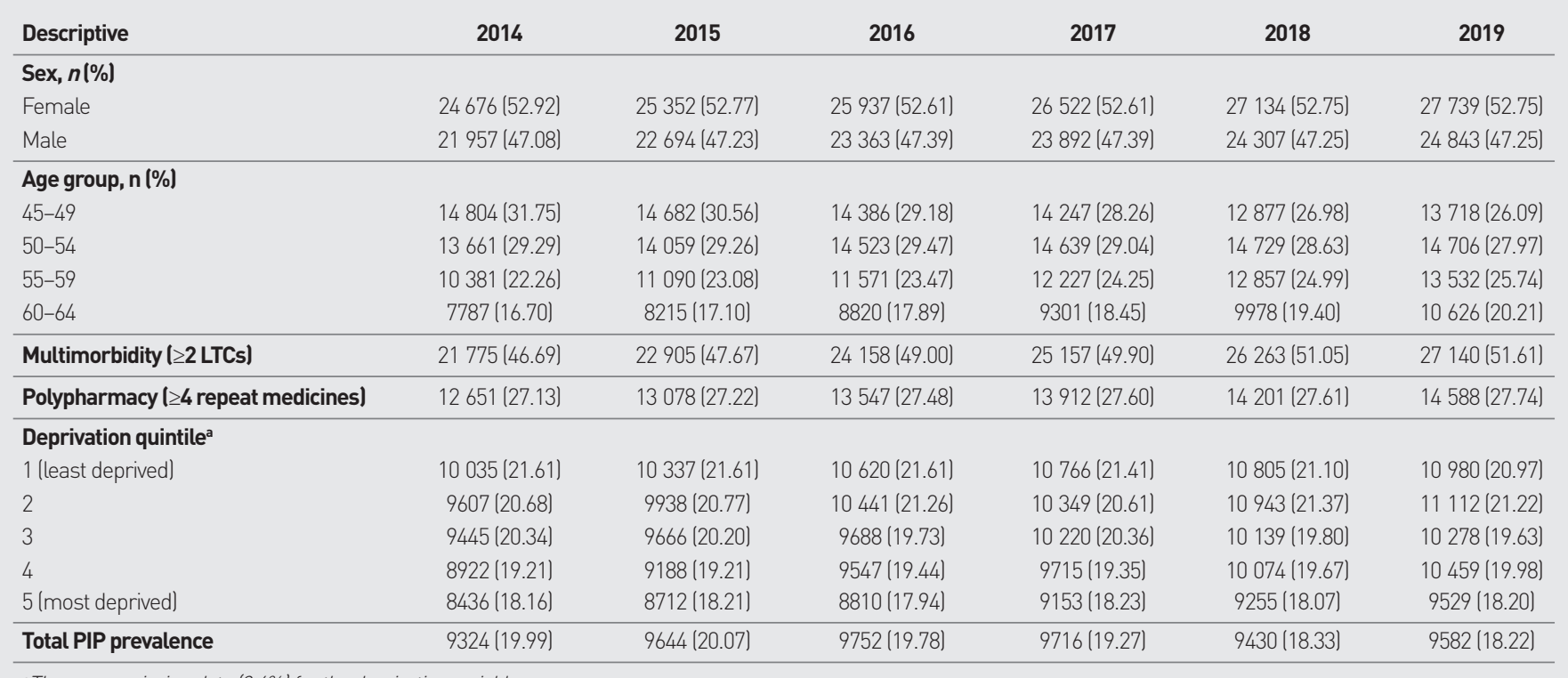

${ }^{a}$ There was missing data $(0.4 \%)$ for the deprivation variable.

Figure 1. Prevalence of polypharmacy, multimorbidity, and PIP over the study period.

PIP = potentially inappropriate prescribing.

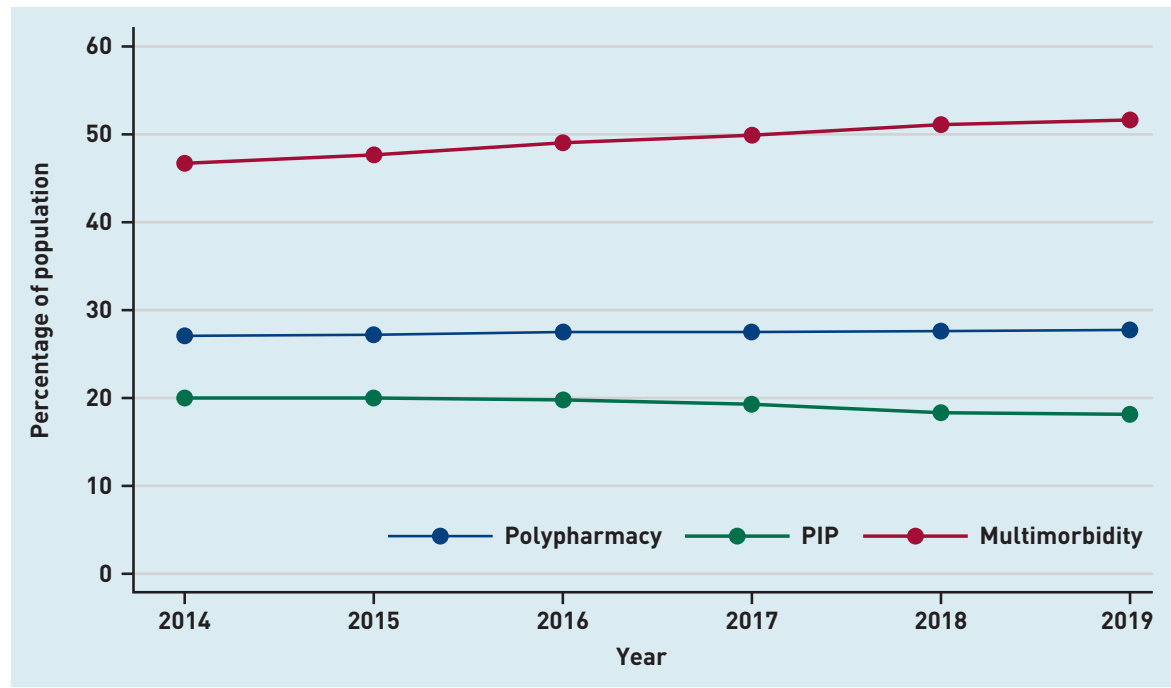

$\sim 12 \%$ ) Between $4-5 \%$ of the population had two and 2-3\% had $\geq 3$ PIPs (see Supplementary Table S2 for details).

\section{Primary outcome}

Percentage prevalence estimates were calculated for each PROMPT criterion (see Supplementary Table S2 for details). Of the 22 criteria, 14 had a percentage prevalence $\leq 0.5 \%$ over the 6 years. The eight most commonly occurring examples of PIP are shown in Figure 2.

In 2014, the most prevalent PROMPT criterion was the use of non-steroidal anti-inflammatory drugs (NSAIDs) for $>3$ months $(n=4458,9.6 \%)$. The prevalence decreased over the 6 years to $7.1 \%$. By 2019 , the concurrent use of $\geq 2$ drugs from the same pharmacological class was the most common criterion ( $n=4003,7.6 \%)$; however, even this represented a decrease from 2014 (8.4\%). The repeated prescription of $\geq 2$ NSAIDs contributed the most to this PROMPT criterion, followed by opioids and selective serotonin reuptake inhibitors (SSRIs) (Figure 3). The use of proton pump inhibitors (PPIs) above the recommended maintenance dosage for $>8$ weeks was the only PROMPT criterion to increase over the study period (from $2.6 \%$ to $3.1 \%$ ).

\section{Analysis}

In the adjusted logistic regression model, a trend of reducing odds of PIP was observed across the study years (Table 2). Polypharmacy was the most strongly associated variable ladjusted odds 


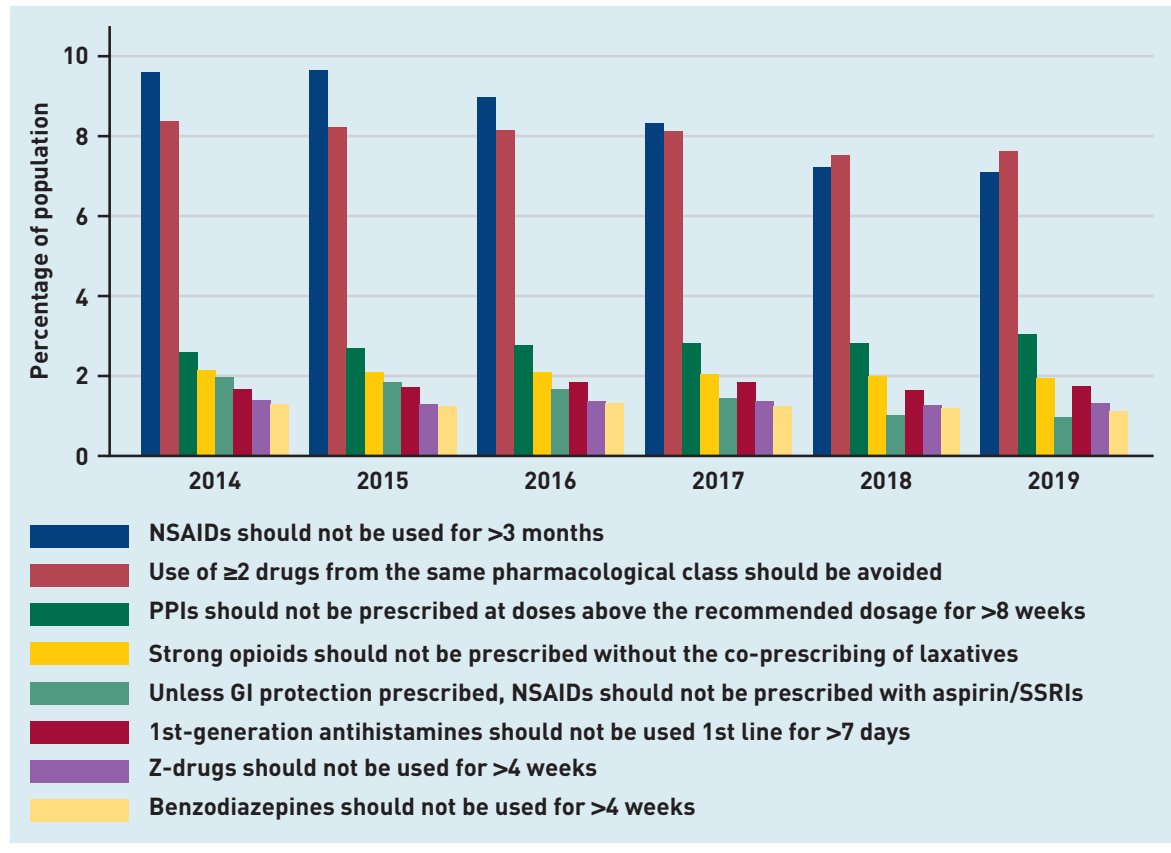

Figure 2. Prevalence of the eight most common PROMPT criteria.

GI = gastrointestinal. NSAID = non-steroidal antiinflammatory drug. $P P I=$ proton pump inhibitor. PROMPT $=$ PRescribing Optimally in Middle-aged People's Treatments. SSRI = selective serotonin reuptake inhibitor.

Figure 3. Prevalence of duplication of drug classes. ACE-I = angiotensin-converting enzyme inhibitor. $A R B=$ angiotensin receptor blocker. $C C B=$ calcium channel blocker. NSAID = non-steroidal antiinflammatory drug. SSRI = selective serotonin reuptake inhibitor. TCA = tricyclic antidepressant.

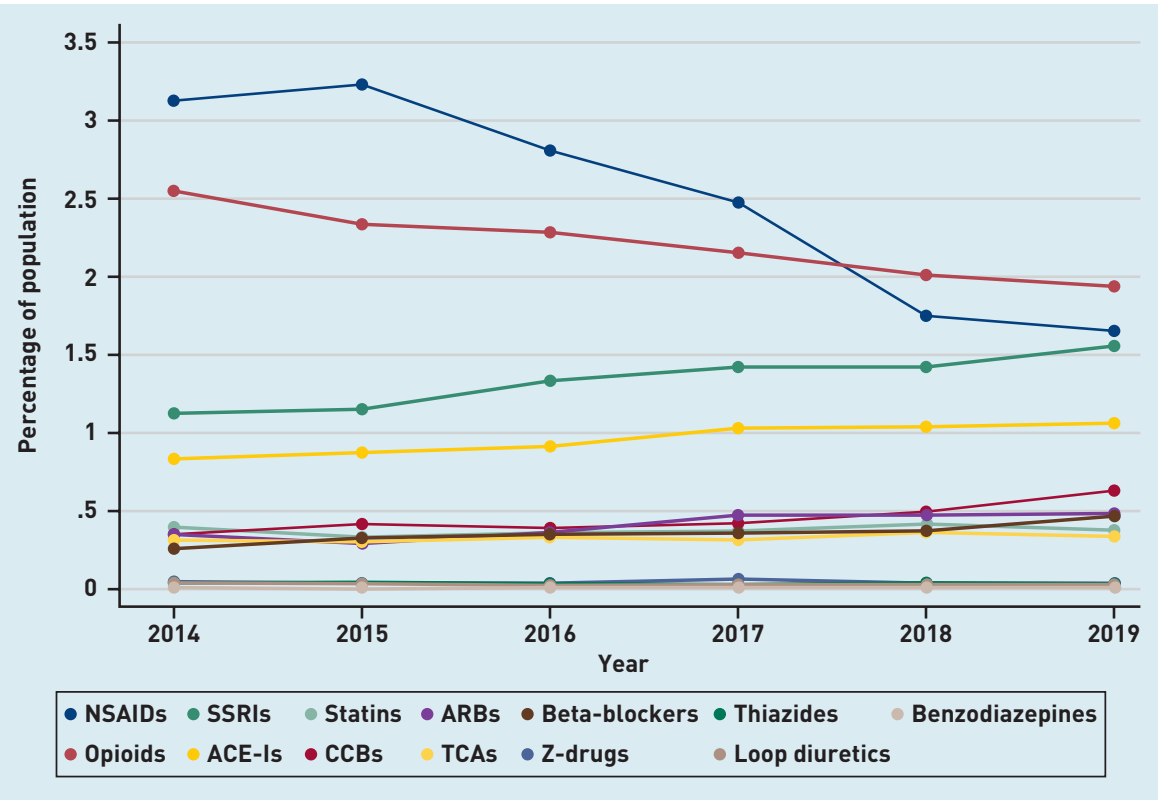

the odds of PIP $(A O R=1.17,95 \% \mathrm{Cl}=1.11$ to 1.23$)$ compared with the least deprived areas. There was no statistically significant association between PIP and female sex (AOR $=1.01,95 \% \mathrm{Cl}=0.97$ to $1.04, P=0.67)$.

Within the negative binomial model, associations of all variables with the rate of PIP were of a similar magnitude and direction, except for female sex. Here, the parameter estimates were analogous to the logistic model, but reached statistical significance in the negative binomial regression $(I R R=1.03,95 \% \quad C l=1.01$ to 1.06, $P=0.02$ ).

The intraclass correlations at the patient and practice level were 0.59 and 0.02 respectively (Table 2), indicating greater correlation between observations from the same patient than among observations from the same general practice.

\section{DISCUSSION}

\section{Summary}

At least $18 \%$ of the study population were prescribed a potentially inappropriate prescription in each year. Across the 6 years of the study, the prevalence of multimorbidity (47-52\%) and polypharmacy (27\%) was high. In 2019, the three most common PIPs were the use of $\geq 2$ drugs concurrently from the same pharmacological class, the use of NSAIDs for $>3$ months, and the use of PPIs above the recommended maintenance dosage for $>8$ weeks. Having polypharmacy $(A O R=4.85, \quad I R R=3.91) \quad$ multimorbidity $(A O R=2.61, \quad I R R=2.47)$, living in a more deprived area, and being older significantly increased the odds and incidence rates of PIP.

\section{Strengths and limitations}

This is the first study to report trends in PIP in middle-aged adults over time. It is also the first to adjust for the clustering of patients and general practices within the regression models and to explore deprivation as an explanatory variable. The use of patient record data (rather than purely prescribing or dispensing datasets) allowed for the examination of additional predictors such as deprivation and multimorbidity. However, patient record data do not include over-the-counter medicines use and may not directly relate to actual dispensing or patient adherence.

Another potential limitation involved the extraction parameter assumptions. It was assumed that the number of prescriptions given equated to a time period for a particular medicine. Therefore, the calculated prevalence for some of the criteria may be underestimated, as some 


\section{Table 2. Logistic and negative binomial regression analyses for PIP}

\begin{tabular}{|c|c|c|c|c|}
\hline \multirow[b]{2}{*}{ Covariate } & \multicolumn{2}{|c|}{ Adjusted logistic regression } & \multicolumn{2}{|c|}{ Negative binomial regression } \\
\hline & OR $(95 \% \mathrm{Cl})$ & $P$-value & IRR (95\% Cl) & $P$-value \\
\hline \multicolumn{5}{|l|}{ Year of study } \\
\hline 2014 (reference) & 1 & - & 1 & - \\
\hline 2015 & 0.99 (0.97 to 1.02 ) & 0.600 & 0.99 (0.97 to 1.01$)$ & 0.256 \\
\hline 2016 & 0.95 (0.93 to 0.98 ) & 0.001 & 0.95 (0.93 to 0.97 ) & $<0.001$ \\
\hline 2017 & 0.90 (0.88 to 0.93 ) & $<0.001$ & 0.91 (0.89 to 0.93 ) & $<0.001$ \\
\hline 2018 & 0.83 (0.80 to 0.86$)$ & $<0.001$ & 0.83 (0.81 to 0.85 ) & $<0.001$ \\
\hline 2019 & 0.81 (0.79 to 0.84 ) & $<0.001$ & 0.82 (0.80 to 0.84 ) & $<0.001$ \\
\hline Polypharmacy & 4.85 (4.69 to 5.02 ) & $<0.001$ & 3.91 (3.80 to 4.03) & $<0.001$ \\
\hline Multimorbidity & 2.61 (2.52 to 2.71 ) & $<0.001$ & 2.47 (2.38 to 2.55 ) & $<0.001$ \\
\hline Female sex & 1.01 (0.97 to 1.04 ) & 0.651 & 1.03 (1.01 to 1.06$)$ & 0.016 \\
\hline \multicolumn{5}{|l|}{ Age group } \\
\hline 45-49 (reference) & 1 & - & 1 & - \\
\hline $50-54$ & 1.05 (1.01 to 1.08$)$ & 0.017 & 1.03 (0.99 to 1.06 ) & 0.111 \\
\hline $55-59$ & 1.10 (1.05 to 1.14$)$ & $<0.001$ & 1.06 (1.02 to 1.09 ) & 0.002 \\
\hline $60-64$ & 1.19 (1.14 to 1.25$)$ & $<0.001$ & 1.11 (1.07 to 1.15 ) & $<0.001$ \\
\hline \multicolumn{5}{|l|}{ Deprivation quintile } \\
\hline 5 (reference, least deprived) & 1 & - & 1 & - \\
\hline 4 & 1.09 (1.03 to 1.15$)$ & 0.002 & 1.09 (1.04 to 1.14$)$ & $<0.001$ \\
\hline 3 & 1.14 (1.08 to 1.20$)$ & $<0.001$ & 1.12 (1.07 to 1.17$)$ & $<0.001$ \\
\hline 2 & 1.18 (1.12 to 1.25$)$ & $<0.001$ & 1.17 (1.12 to 1.23 ) & $<0.001$ \\
\hline 1 (most deprived) & 1.17 (1.11 to 1.23$)$ & $<0.001$ & $1.16(1.11$ to 1.21$)$ & $<0.001$ \\
\hline
\end{tabular}

$\mathrm{Cl}=$ confidence interval. $\mathrm{OR}=$ odds ratio. $I R R=$ incidence rate ratio. the findings is supported by the prevalence of polypharmacy $(27 \%)$ being consistent with existing studies. ${ }^{14,22}$

\section{Comparison with existing literature}

The literature analysing PIP in middle-aged adults is very limited. Only four previous studies measured the prevalence of PIP using the PROMPT criteria. ${ }^{12,14-16}$ This study reported a lower prevalence of PIP (18\%) compared with previous studies (ranging from 21.1-42.9\%). Harasani and others ${ }^{15}$ looked at the dispensing data of a selection of community pharmacies in Albania, and Moriarty and others ${ }^{14}$ analysed participants from a means-tested scheme in Ireland, with the representativeness of these studies limited owing to their selected population.

This study found that the use of $\geq 2$ drugs concurrently from the same pharmacological class was one of the most prevalent PIPs. Previous studies reported that NSAIDs and opioids are commonly implicated in this criterion, similar to this study. ${ }^{12,14}$ In contrast to previous studies, the prescribing of duplicates of benzodiazepines was found to be negligible. Furthermore, the use of NSAIDs for $>3$ months and the use of PPIs above the recommended maintenance dosage for $>8$ weeks commonly occurred, as in existing studies. $12,14,15$

Research has consistently shown a positive association between PIP and polypharmacy, in both middle-aged and older adults. ${ }^{12,23}$ The relationship between multimorbidity and PIP in middle-aged adults is less well understood; however, when it has been included in previous studies, it also shows a positive association with PIP. ${ }^{14,24}$ Both female sex and increasing age are inconsistently associated with PIP in older adults, but studies in middle-aged adults found a positive association. ${ }^{12,14,23}$ The observed increased odds of PIP with greater deprivation confirms the arguments raised by Cooper and others ${ }^{12}$ in their comparative study of two populations differing in their deprivation levels.

\section{Implications for research and practice}

It is known that ADEs are associated with PIP in middle-aged adults. ${ }^{16}$ This study provides evidence that the prevalence of PIP in middle-aged adults is high. Therefore, intervening to optimise prescribing in this age group may reduce these ADEs.

National Institute for Health and Care Excellence guidelines on medicines optimisation advise using screening tools to monitor PIP in older adults and those with polypharmacy. ${ }^{25}$ The results of this study underline that PIP is not confined to 


\section{Funding}

None.

\section{Ethical approval}

All data were extracted under the terms of a signed data-sharing agreement with each practice and with project-specific approval following submission of a data privacy impact assessment, approved by Lambeth Clinical Commissioning Group on 2 November 2017. Information governance approval required 'low number suppression', ensuring that data could not be displayed if the patient number was 10 or less in any given category; in these circumstances, data reporting would state: ' $\leq 10$ patients'. Separate ethical committee approval was not required (Health Research Authority, personal correspondence, 29 September 2017) since all data were fully anonymised for the purposes of research access, and all patient identifiable data had been removed. ${ }^{33}$

\section{Provenance}

Freely submitted; externally peer reviewed.

\section{Competing interests}

Patrick Redmond is funded by the National Institute for Health Research (NIHR) as an Academic Clinical Lecturer. The views and opinions expressed by authors are those of the authors and do not necessarily reflect those of the UK National Institute for Health Research (NIHR) or the Department of Health and Social Care.

\section{Acknowledgements}

We would like to acknowledge the contribution of the patients and practices of Lambeth for granting us access to their data.

\section{Open access}

This article is Open Access: CC BY 4.0 licence (http://creativecommons.org/ licences/by/4.0/).

\section{Discuss this article}

Contribute and read comments about this article: bjgp.org/letters older adults, and so there should also be a specific focus in the guidelines on the role of tools such as PROMPT in middle-aged adults.

Currently, prescribing optimisation interventions are primarily focused on older adults, and aim to increase awareness of PIP and improve prescribing appropriateness. ${ }^{26,27}$ Given the common nature of PIP in middle-aged adults, future research should investigate the benefit of primary care-based interventions to improve prescribing in this group also. For example, clinical decision support (CDS) tools could incorporate the PROMPT criteria. However, CDS tools are known to have limitations, notably that prescribers can override and ignore them. ${ }^{28,29}$
PIP of NSAIDs and PPIs relate to longterm use, which could be addressed through frequent medication reviews. A metaanalysis of medicine reviews has found that they can reduce PIP-related ADEs. ${ }^{30}$ Given the coexistence of PIP, multimorbidity, polypharmacy, and deprivation, targeting the delivery of these interventions may improve their effectiveness. This may be supported by future research further delineating disease patterns most at risk of PIP. However, reviews can be time consuming and specialised, requiring careful consideration of risk/ benefit profiles. Increasingly, the role of practice-based pharmacists in performing reviews is recognised, ${ }^{31}$ allowing more thorough reviews and increased patient participation. $^{32}$ 


\section{REFERENCES}

1. O'Mahony D, Gallagher PF. Inappropriate prescribing in the older population: need for new criteria. Age Ageing 2008; 37(2): 138-141.

2. Liew TM, Lee CS, Goh KL, et al. Potentially inappropriate prescribing among older persons: a meta-analysis of observational studies. Ann Fam Med 2019; 17(3): 257-266.

3. Black CD, Thavorn K, Coyle D, et al. Health system costs of potentially inappropriate prescribing in Ontario, Canada: a protocol for a population-based cohort study. BMJ Open 2018; 8(6): e021727.

4. Guthrie B, Donnan PT, Murphy DJ, et al. Bad apples or spoiled barrels? Multilevel modelling analysis of variation in high-risk prescribing in Scotland between general practitioners and between the practices they work in. BMJ Open 2015; 5(11): e008270.

5. Opondo D, Eslami S, Visscher S, et al. Inappropriateness of medication prescriptions to elderly patients in the primary care setting: a systematic review. PLoS One; 7(8): e43617.

6. Masnoon N, Shakib S, Kalisch-Ellett L, et al. What is polypharmacy? A systematic review of definitions. BMC Geriatr 2017; 17(1): 230.

7. Al-Busaidi S, Al-Kharusi A, Al-Hinai M, et al. Potentially inappropriate prescribing among elderly patients at a primary care clinic in Oman. J Cross Cult Gerontol 2020; 35(2): 209-216.

8. Payne RA, Avery AJ, Duerden M, et al. Prevalence of polypharmacy in a Scottish primary care population. Eur J Clin Pharmacol 2014; 70(5): 575-581.

9. Payne RA. The epidemiology of polypharmacy. Clin Med (Lond) 2016; 16(5): 465-469

10. Johnston MC, Crilly M, Black C, et al. Defining and measuring multimorbidity: a systematic review of systematic reviews. Eur J Public Health 2018; 29(1): 182-189.

11. Barnett $K$, Mercer SW, Norbury M, et al. Epidemiology of multimorbidity and implications for health care, research, and medical education: a crosssectional study. Lancet 2012; 380(9836): 37-43.

12. Cooper JA, Moriarty F, Ryan C, et al. Potentially inappropriate prescribing in two populations with differing socio-economic profiles: a cross-sectional database study using the PROMPT criteria. Eur J Clin Pharmacol 2016; 72(5): 583-591.

13. Cooper JA, Ryan C, Smith SM, et al. The development of the PROMPT (PRescribing Optimally in Middle-aged People's Treatments) criteria. BMC Health Serv Res 2014; 14: 484

14. Moriarty F, Cahir C, Bennett $\mathrm{K}$, et al. Potentially inappropriate prescribing and its association with health outcomes in middle-aged people: a prospective cohort study in Ireland. BMJ Open 2017; 7(10): e016562.

15. Harasani K, Xhafaj D, Qipo O. Prevalence and types of potentially inappropriate prescriptions among older and middle-aged community-dwelling Albanian patients. Int J Risk Saf Med 2020; 31(1): 5-13.

16. Smeaton T, McElwaine $P$, Cullen J, et al. A prospective observational pilot study of adverse drug reactions contributing to hospitalization in a cohort of middleaged adults aged 45-64 years. Drugs Ther Perspect 2020; 36: 123-130.

17. Von Elm E, Altman D, Egger M, et al. The Strengthening the Reporting of Observational Studies in Epidemiology (STROBE) Statement: guidelines for reporting observational studies. J Clin Epidemiol 2008; 61(4): 344-349.
18. Rao R, Schofield P, Ashworth M. Alcohol use, socioeconomic deprivation and ethnicity in older people. BMJ Open 2015; 5(8): e007525.

19. Dorrington S, Carr E, Stevelink SAM, et al. Demographic variation in fit note receipt and long-term conditions in south London. Occup Environ Med 2020; 77(6): 418-426.

20. Cassell A, Edwards D, Harshfield A, et al. The epidemiology of multimorbidity in primary care: a retrospective cohort study. Br J Gen Pract 2018; DOI: https://doi. org/10.3399/bjgp18X695465

21. Fick DM, Semla TP, Steinman M, et al. American Geriatrics Society 2019 Updated AGS Beers Criteria ${ }^{\circledR}$ for Potentially Inappropriate Medication Use in Older Adults. J Am Geriatr Soc 2019; 67(4): 674-694.

22. Moriarty F, Hardy C, Bennett K, et al. Trends and interaction of polypharmacy and potentially inappropriate prescribing in primary care over 15 years in Ireland: a repeated cross-sectional study. BMJ Open 2015; 5(9): e008656.

23. Tommelein E, Mehuys E, Petrovic M, et al. Potentially inappropriate prescribing in community-dwelling older people across Europe: a systematic literature review. Eur J Clin Pharmacol 2015; 71(12): 1415-1427.

24. Pérez T, Moriarty F, Wallace E, et al. Prevalence of potentially inappropriate prescribing in older people in primary care and its association with hospital admission: longitudinal study. BMJ 2018; 363: k4524.

25. National Institute for Health and Care Excellence. Medicines optimisation: the safe and effective use of medicines to enable the best possible outcomes. NG5. London: NICE, 2015. https://www.nice.org.uk/guidance/ng5 laccessed 4 Mar 2021).

26. Gillespie P, Clyne B, Raymakers A, et al. Reducing potentially inappropriate prescribing for older people in primary care: cost-effectiveness of the optiscript intervention. Int J Technol Assess Health Care 2017; 33(4): 494-503.

27. Riordan DO, Walsh KA, Galvin R, et al. The effect of pharmacist-led interventions in optimising prescribing in older adults in primary care: a systematic review. SAGE Open Med 2016; 4: 2050312116652568.

28. Lapane KL, Waring ME, Schneider KL, et al. A mixed method study of the merits of e-prescribing drug alerts in primary care. J Gen Intern Med 2008; 23(4): 442-446.

29. Slight SP, Nanji KC, Seger DL, et al. Overrides of clinical decision support alerts in primary care clinics. Stud Health Technol Inform 2013; 192: 923.

30. Huiskes VJB, Burger DM, van den Ende CHM, et al. Effectiveness of medication review: a systematic review and meta-analysis of randomized controlled trials. BMC Fam Pract 2017; 18(1): 5.

31. Duncan P, Cabral C, Mccahon D, et al. Efficiency versus thoroughness in medication review: a qualitative interview study in UK primary care. $\mathrm{Br} J \mathrm{Gen}$ Pract 2019; DOI: https://doi.org/10.3399/bjgp19X701321.

32. Uhl MC, Muth C, Gerlach FM, et al. Patient-perceived barriers and facilitators to the implementation of a medication review in primary care: a qualitative thematic analysis. BMC Fam Pract 2018; 19(1): 3.

33. Ashworth M, Durbaba S, Whitney D, et al. Journey to multimorbidity: longitudinal analysis exploring cardiovascular risk factors and sociodemographic determinants in an urban setting. BMJ Open 2019; 9(12): e031649. 